This is a postprint version of the following published document:

Marcellán, F., Mendes, A., \& Pijeira, H. (2013). Bases of the space of solutions of some fourth-order linear diference equations : applications in rational approximation. Journal of Difference Equations and Applications, 19, pp. 1632-1644

DOI: $\underline{10.1080 / 10236198.2013 .769531}$

Proyecto: MTM2012- 36732- C03-01

(C) Taylor \& Francis 2013 


\title{
Bases of the space of solutions of some fourth-order linear difference equations. Applications in rational approximation.
}

\author{
Francisco Marcellán ${ }^{\dagger \ddagger}$, Ana Mendes ${ }^{\natural}$ and Héctor Pijeira ${ }^{\dagger \ddagger}$ \\ ${ }^{\dagger}$ Departamento de Matemáticas, Universidad Carlos III de Madrid, Spain; ${ }^{\natural}$ Escola \\ Superior de Tecnologia e Gestão, Instituto Politécnico de Leiria, Portugal.
}

(Received 00 Month 20xx; in final form 00 Month 20xx)

It is very well known that a sequence of polynomials $\left\{Q_{n}(x)\right\}_{n=0}^{\infty}$ orthogonal with respect to a Sobolev discrete inner product

$$
\langle f, g\rangle_{S}=\int_{I} f g d \mu+\lambda f^{\prime}(0) g^{\prime}(0), \quad \lambda \in \mathbb{R}^{+},
$$

where $\mu$ is a finite Borel measure and $I$ is an interval of the real line, satisfies a five-term recurrence relation.

In this contribution we study other three families of polynomials which are linearly independent solutions of such a five term linear difference equation and, as a consequence, we obtain a polynomial basis of such a linear space. They constitute the analog of the associated polynomials in the standard case. Their explicit expression in terms of $\left\{Q_{n}(x)\right\}_{n=0}^{\infty}$ using an integral representation is given. Finally, an application of these polynomials in rational approximation is shown.

Keywords: orthogonal polynomials, recurrence relation, difference equations.

AMS Subject Classification: Primary 42C05 ; Secondary 33C25.

\section{Introduction}

Let $\mu$ be a positive finite Borel measure supported on the real line and suppose that $I=\operatorname{supp}(\mu)$ is an infinite set of points. Let us define the inner product

$$
\langle f, g\rangle_{\mu}:=\int_{I} f(x) g(x) d \mu(x),
$$

and the corresponding norm $\|f\|_{\mu}=\sqrt{\langle f, f\rangle_{\mu}}$. Obviously, (1) satisfies the identity

$$
\langle x f(x), g(x)\rangle_{\mu}=\langle f(x), x g(x)\rangle_{\mu},
$$

i.e. the multiplication operator is symmetric with respect to the above inner product.

If $\left\{P_{n}(x)\right\}_{n=0}^{\infty}$ is the sequence of monic orthogonal polynomials with respect to the inner product (1), a direct consequence of (2) is that $\left\{P_{n}(x)\right\}_{n=0}^{\infty}$ satisfies a three term recurrence relation

$$
P_{n+1}(x)=\left(x-\eta_{n}\right) P_{n}(x)-\gamma_{n} P_{n-1}(x), \quad n \geq 0,
$$

\footnotetext{
*Corresponding author. Email: pacomarc@ing.uc3m.es

${ }^{\ddagger}$ Research partially supported by Dirección General de Investigación Científica y Técnica, Ministerio de Economía y Competitividad of Spain, under grant MTM2012-36732-C03-01.
} 


$$
\eta_{n}=\frac{1}{\left\|P_{n}\right\|_{\mu}^{2}} \int_{I} x P_{n}^{2}(x) d \mu(x), \gamma_{n}=\frac{\left\|P_{n}\right\|_{\mu}^{2}}{\left\|P_{n-1}\right\|_{\mu}^{2}}, \gamma_{0}=|\mu|=\mu(I),
$$

with initial conditions $P_{-1}(x)=0$ and $P_{0}(x)=1$.

Equivalently, we can rewrite (3) in terms of a linear difference equation of order two as

$$
\tau_{n+1} Y_{n+1}=\left(x-\eta_{n}\right) Y_{n}-\tau_{n} Y_{n-1}, \quad \tau_{n}^{2}=\gamma_{n}, n \geq 0
$$

with initial conditions $Y_{-1}=0$ and $Y_{0}=1$. It is well known that the set of solutions of (4) is a bidimensional linear space. Of course one solution is $\left\{p_{n}\right\}_{n=0}^{\infty}$ where $p_{n}=\left\|P_{n}\right\|_{\mu}^{-1} P_{n}(x)$. Another linearly independent solution, is the (not necessarily monic) polynomial $p_{n-1}^{[1]}\left(\operatorname{deg}\left(p_{n-1}^{[1]}\right)=n-1\right)$ that can be obtained from (4) with the initial conditions $Y_{-1}=1$ and $Y_{0}=0$. In the theory of orthogonal polynomials the sequence $\left\{p_{n}^{[1]}\right\}_{n=0}^{\infty}$ is often called sequence of first associated, numerator or second kind polynomials with respect to the sequence of monic orthogonal polynomials $\left\{P_{n}\right\}_{n=0}^{\infty}$.

The recursion (3) plays an important role in the study of analytic and algebraic properties of orthogonal polynomials. In fact, this three term recurrence relation characterizes the orthogonality with respect to a measure. This result is known in the literature as Favard theorem (see [5]) and states that if a sequence of monic polynomials $\left\{P_{n}\right\}_{n=0}^{\infty}$ satisfies a three-term recurrence relation as (3), with $\eta_{n} \in \mathbb{R}$ and $\gamma_{n} \in \mathbb{R}_{+}$, then there exists a positive Borel measure $\mu$ such that $\left\{P_{n}\right\}_{n=0}^{\infty}$ is orthogonal with respect to the inner product (1).

Note that $P_{n}^{[1]}=|\mu|^{-1} p_{n}^{[1]}$ is a sequence of monic polynomials satisfying the three term recurrence relation

$$
P_{n+1}^{[1]}(x)=\left(x-\eta_{n+1}\right) P_{n}^{[1]}(x)-\gamma_{n+1} P_{n-1}^{[1]}(x), \quad n \geq 0,
$$

with initial conditions $P_{-1}^{[1]}=0$ and $P_{0}^{[1]}=1$. According to the Favard theorem there exists a positive Borel measure $\nu$ such that $\left\{P_{n}^{[1]}\right\}_{n=0}^{\infty}$ is orthogonal with respect to the inner product

$$
\langle f, g\rangle_{\nu}:=\int_{I} f(x) g(x) d \nu(x)
$$

(see [12]).

It is well known that $\left\{P_{n}\right\}$ and $\left\{P_{n}^{[1]}\right\}$ satisfy

(1) $P_{n}^{[1]}(z)=\frac{1}{|\mu|} \int_{I} \frac{P_{n+1}(z)-P_{n+1}(x)}{z-x} d \mu(x)$.

(2) If $I$ is a bounded interval, $\frac{P_{n-1}^{[1]}(z)}{P_{n}(z)} \underset{n \rightarrow \infty}{\rightrightarrows} \frac{1}{|\mu|} \int_{I} \frac{d \mu}{z-x}$, uniformly on compact subsets of $\mathbb{C} \backslash \operatorname{supp}(\mu)$ (Markov theorem).

(3) $\nu$ is an absolutely continuous measure with respect to $\mu$ (see [9, Theorem 3]).

(4) Every solution of (4) can be written as $y_{n}=A(x) P_{n}(x)+B(x) P_{n-1}^{[1]}(x)$, where $A(x)$ and $B(x)$ are functions which can be explicitly given in terms of the initial conditions of (4).

In [2] the author proved that a sequence of polynomials satisfying a higher order recurrence relation is closely related to a sequence of matrix polynomials satisfying a 
three term recurrence relation and that Favard's theorem for matrix polynomials and Favard's theorem for polynomials satisfying such a higher order recurrence relation are the same. Indeed, if $\left\{r_{n}\right\}_{n=0}^{\infty}$ is a sequence of polynomials satisfying a recurrence relation like $x^{N} r_{n}(x)=c_{n, 0} r_{n}(x)+\sum_{k=1}^{N}\left(c_{n, k} r_{n-k}(x)+c_{n+k, k} r_{n-k}(x)\right)$ where $\left(c_{n, N}\right)_{n=0}^{\infty}$ is a nonvanishing real sequence and $\left(c_{n, k}\right)_{n=0}^{\infty}$, with $0 \leq k \leq N-1$, are real numbers, assuming, as a convention, that if $k<0$ then $c_{n, k}=r_{k}(x)=0$. Notice that for $N=1$, we get the three term recurrence relation (3). It is easy to prove that if the multiplication operator $T_{N}$ defined in the linear space of polynomials $\mathbf{P}$ by $\mathrm{T}_{N} p(x)=x^{N} p(x)$ is selfadjoint for an inner product $B$, then the set of orthonormal polynomials with respect to $B$ satisfies a $2 N+1$ recurrence relation. In [2] the integral representation for such an inner product is deduced. A characterization of general (non diagonal) discrete Sobolev inner products is given in an elegant way using that in such a case $B\left(\mathrm{~T}_{N} p(x), \mathrm{T}_{1} q(x)\right)=B\left(\mathrm{~T}_{1} p(x), \mathrm{T}_{N} q(x)\right)$. In particular, the diagonal case appears if and only if the extra condition $B\left(x^{k}, x^{m}\right)=B\left(1, x^{m+k}\right)$, when $1 \leq k, m \leq N-1$ and $k \neq m$, holds.

Later on, in [3], by considering a general polynomial multiplication operator, the authors show that discrete Sobolev orthogonal polynomials are related to orthogonal matrix polynomials and they obtain the orthogonality matrix measure in terms of the parameters in the inner product. The advantage of this approach using matrix orthogonal polynomials is that the orthogonality conditions no longer require the evaluation of a function and their derivatives at several points.

On the other hand, in [4] the authors characterized discrete Sobolev inner products using another approach. The support of the discrete part of such an inner product is also deduced.

The aim of this paper is to analyze the set of solutions of the fourth order linear difference equation defined by the above five term recurrence relation when $N=2$ and we assume that the bilinear functional $B$ is defined by a particular case of discrete Sobolev inner product. We obtain a polynomial basis of such a linear subspace using a generalization of associated polynomials of order $k$ with $k=1,2,3$, for the sequence of discrete Sobolev orthonormal polynomials. This approach is quite different of the presented in [8] based on the generalization of the Taylor expansion of the above orthonormal polynomials.

The structure of the manuscript is as follows. In Section 2 we consider the five term recurrence relation that a sequence of monic polynomials, orthogonal with respect to a discrete Sobolev inner product, satisfies. We obtain a basis for the space of solutions of the corresponding fourth order linear difference equation. In Section 3, the location of the zeros of such orthogonal polynomials is deduced and we emphasize in their interlacing properties. Section 4 is focused on Gauss Quadrature formulas associated with the zeros of such orthogonal polynomials. Finally, in Section 5 we obtain some convergence results for a sequence of rational functions whose denominators are the above discrete Sobolev orthogonal polynomials. An estimate of their speed of convergence is given. 


\section{Five-term recurrence relations}

Let $\mu$ be a measure with the same properties as above and let introduce the Sobolev inner product

$$
\langle f, g\rangle_{S}:=\int_{I} f(x) g(x) d \mu(x)+\lambda f^{\prime}(0) g^{\prime}(0), \quad \text { where } \quad \lambda \in \mathbb{R}^{+},
$$

and the corresponding norm $\|f\|_{S}=\sqrt{\langle f, f\rangle_{S}}$. Again it is straightforward to prove that

$$
\left\langle x^{2} f, g\right\rangle_{S}=\left\langle f, x^{2} g\right\rangle_{S}
$$

Hence, the sequence of monic orthogonal polynomials with respect to $(7),\left\{Q_{n}\right\}_{n=0}^{\infty}$, satisfies a five term recurrence relation (see [1] and [11]). Notice that this is a particular case of the problem analyzed in [7], where recurrence relations for polynomials orthogonal with respect to the discrete Sobolev inner product involving the $k$ th order derivative at the point $x=0$

$$
\langle f, g\rangle_{S}:=\int_{I} f(x) g(x) d \mu(x)+\lambda f^{(k)}(0) g^{(k)}(0), \quad \text { where } \quad \lambda \in \mathbb{R}^{+},
$$

are studied.

Indeed,

$$
Q_{n+2}(x)=\left(x^{2}-\beta_{n, 0}\right) Q_{n}(x)-\beta_{n, 1} Q_{n+1}(x)-\beta_{n,-1} Q_{n-1}(x)-\alpha_{n}^{2} Q_{n-2}(x),
$$

with $n \geq 2$, and the initial conditions

$$
\begin{aligned}
Q_{-2}(x) & =0, Q_{-1}(x)=0, \\
Q_{0}(x) & =1, \quad Q_{1}(x)=P_{1}(x)=x-a,
\end{aligned}
$$

where

$$
a=\frac{1}{|\mu|} \int_{I} x d \mu(x), \alpha_{n}=\frac{\left\|Q_{n}\right\|_{S}}{\left\|Q_{n-2}\right\|_{S}}, \text { and } \beta_{n, k}=\frac{\left\langle x^{2} Q_{n}, Q_{n+k}\right\rangle_{S}}{\left\|Q_{n+k}\right\|_{S}^{2}}, \quad k=-1,0,1 .
$$

Let $\left\{R_{n}\right\}_{n=0}^{\infty}$ be the sequence of monic orthogonal polynomials with respect to the measure $d \mu_{2}(x)=x^{2} d \mu(x)$. We define for $k \in \mathbb{N}$ the $k$ th associated polynomials $\left\{R_{n}^{[k]}\right\}_{n=0}^{\infty}$ by the recurrence relations

$$
\begin{gathered}
R_{n+1}^{[k]}(x)=\left(x-\eta_{n+k, 2}\right) R_{n}^{[k]}(x)-\gamma_{n+k, 2} R_{n-1}^{[k]}(x), \\
\eta_{n+k, 2}=\frac{1}{\left\|R_{n+k}\right\|_{\mu_{2}}^{2}} \int_{I} x R_{n+k}^{2}(x) d \mu_{2}(x), \gamma_{n+k, 2}=\frac{\left\|R_{n+k}\right\|_{\mu_{2}}^{2}}{\left\|R_{n+k-1}\right\|_{\mu_{2}}^{2}}, \gamma_{0,2}=\left|\mu_{2}\right|=\mu_{2}(I),
\end{gathered}
$$

with initial conditions $R_{-1}^{[k]}=0, R_{0}^{[k]}=1$. Furthermore, the sequences of $k$ th associated polynomials can be represented by the following formula (see $[12,(2.13)]$ )

$$
R_{n}^{[k]}(x)=\frac{1}{m_{k}} \int_{I} \frac{R_{n+k}(x)-R_{n+k}(t)}{x-t} R_{k-1}(t) d \mu_{2}(t), \text { for } k \in \mathbb{N} \text { and } n \geq 2,
$$

where $m_{k}=\int_{I} R_{k-1}^{2}(t) d \mu_{2}(t)$. Additionally, by $R_{n}^{[0]}(x)$ we denote the polynomial $R_{n}(x)$. The next formula expresses the well-known relationship between $k$ th associated polynomials

$$
R_{n-k}^{[k+1]}(x) R_{n-k}^{[k]}(x)-R_{n-k+1}^{[k]}(x) R_{n-k-1}^{[k+1]}(x)=\prod_{i=1}^{n-k} \gamma_{k+i, 2}=\frac{\left\|R_{n}\right\|_{\mu_{2}}^{2}}{\left\|R_{k}\right\|_{\mu_{2}}^{2}}>0 .
$$


From the standard theory of orthogonal polynomials it is well known that a direct consequence of (11) and (12) is that the polynomial $R_{n}^{[k]}$ does not have common zeros with the polynomials $R_{n-1}^{[k]}$ and $R_{n}^{[k+1]}$. Furthermore, from [12, (2.5)]

$$
R_{n}^{[i-1]}(x)=\left(x-\eta_{i-1,2}\right) R_{n-1}^{[i]}(x)-\gamma_{i, 2} R_{n-2}^{[i+1]}(x) .
$$

Now, we associate to the sequence $\left\{Q_{n}\right\}_{n=0}^{\infty}$ the next three sequences of polynomials

$$
Q_{n}^{[i]}(x)=\frac{1}{m_{i}} \int_{I} \frac{Q_{n+i}(x)-Q_{n+i}(t)}{x-t} R_{i-1}(t) d \mu_{2}(t),
$$

for $i=1,2,3$ and $n \geq 1$. Additionally, by $Q_{n}^{[0]}(x)$ we denote the polynomial $Q_{n}(x)$. Note that $Q_{n}^{[i]}$ is a monic polynomial of degree $n$, for $i=1,2,3$.

THEOREM 2.1. For $n \geq 3$, the sequences of monic polynomials $\left\{Q_{n}^{[i]}\right\}_{n=0}^{\infty}$, where $i=0,1,2,3$, satisfy the following five term recurrence relations

$$
\begin{aligned}
Q_{n+2}^{[i]}(x)= & \left(x^{2}-\beta_{n+i, 0}\right) Q_{n}^{[i]}(x)-\beta_{n+i, 1} Q_{n+1}^{[i]}(x)-\beta_{n+i,-1} Q_{n-1}^{[i]}(x) \\
& -\alpha_{n+i}^{2} Q_{n-2}^{[i]}(x),
\end{aligned}
$$

respectively, with initial conditions $Q_{-2}^{[i]}=Q_{-1}^{[i]}=0, Q_{0}^{[i]}=1, i=0,1,2,3$, and

$$
\begin{aligned}
Q_{1}^{[0]}(x) & =P_{1}(x), \\
Q_{1}^{[i]}(x) & =\frac{1}{m_{i}} \int_{I} \frac{Q_{i+1}(x)-Q_{i+1}(t)}{x-t} R_{i-1}(t) d \mu_{2}(t) \quad \text { for } i=1,2,3 .
\end{aligned}
$$

Proof. For $i=0$ the recurrence relations (15) and (10) are the same. Suppose that $i=1,2$ or 3 , hence by $(10)$

$$
\begin{aligned}
x^{2} Q_{n+i}(x)= & Q_{n+i+2}(x)+\beta_{n+i, 1} Q_{n+i+1}(x)+\beta_{n+i, 0} Q_{n+i}(x) \\
& +\beta_{n+i,-1} Q_{n+i-1}(x)+\alpha_{n+i}^{2} Q_{n+i-2}(x), \\
t^{2} Q_{n+i}(t)= & Q_{n+i+2}(t)+\beta_{n+i, 1} Q_{n+i+1}(t)+\beta_{n+i, 0} Q_{n+i}(t) \\
& +\beta_{n+i,-1} Q_{n+i-1}(t)+\alpha_{n+i}^{2} Q_{n+i-2}(t) .
\end{aligned}
$$

Subtracting these two relations, multiplying both sides of the equation by $\frac{R_{i-1}(t)}{m_{i}(x-t)}$ and integrating on $I$ with respect to $d \mu_{2}(t)$, we get

$$
\begin{aligned}
& \int_{I} \frac{x^{2} Q_{n+i}(x)-t^{2} Q_{n+i}(t)}{m_{i}(x-t)} R_{i-1}(t) d \mu_{2}(t) \\
& =Q_{n+2}^{[i]}(x)+\beta_{n+i, 1} Q_{n+1}^{[i]}(x)+\beta_{n+i, 0} Q_{n}^{[i]}(x)+\beta_{n+i,-1} Q_{n-1}^{[i]}(x)+\alpha_{n+i}^{2} Q_{n-2}^{[i]}(x) .
\end{aligned}
$$

Adding and subtracting $x^{2} Q_{n+i}(t)$ in the numerator of the left side of the last equation and using the orthogonality condition, for $n \geq 3$ we get

$$
\frac{1}{m_{i}} \int_{I} \frac{x^{2} Q_{n+i}(x)-t^{2} Q_{n+i}(t)}{x-t} R_{i-1}(t) d \mu_{2}(t)=x^{2} Q_{n}^{[i]}(x) .
$$

Now, we recall the initial problem of this section, find a basis for the linear space of the polynomial solutions of the recurrence relation (10). This recurrence relation can be interpreted as a linear difference equation of fourth order in the following way:

$$
Y_{n+2}=\left(x^{2}-\beta_{n, 0}\right) Y_{n}-\beta_{n, 1} Y_{n+1}-\beta_{n,-1} Y_{n-1}-\alpha_{n}^{2} Y_{n-2}, \quad n \geq 2,
$$


where $x$ is the parameter and $Y_{n}: \mathbb{N} \rightarrow \mathbf{P}$ is a polynomial solution of (16). Let $\mathcal{S}$ the linear space of polynomial solutions of (16) with dimension 4. Consider the following systems of polynomials

$$
Y_{n, 0}=Q_{n}^{[0]}(x), \quad Y_{n, 1}=Q_{n-1}^{[1]}(x), \quad Y_{n, 2}=Q_{n-2}^{[2]}(x) \quad \text { and } \quad Y_{n, 3}=Q_{n-3}^{[3]}(x) .
$$

THEOREM 2.2. The set of the four sequences $\left\{Y_{n, 0}\right\}_{n=0}^{\infty},\left\{Y_{n, 1}\right\}_{n=0}^{\infty},\left\{Y_{n, 2}\right\}_{n=0}^{\infty}$, $\left\{Y_{n, 3}\right\}_{n=0}^{\infty}$ is a basis of $\mathcal{S}$.

Proof. From Theorem 2.1 it is straightforward to prove that $\left\{Y_{n, k}\right\}_{n=0}^{\infty}, k=0,1,2,3$, are solutions of (16), with initial conditions

$$
\begin{aligned}
& Y_{0,0}=1, Y_{1,0}=Q_{1}(x), Y_{2,0}=Q_{2}(x), Y_{3,0}=Q_{3}(x) \text {. } \\
& Y_{0,1}=0, Y_{1,1}=1, \quad Y_{2,1}=Q_{1}^{[1]}(x), Y_{3,1}=Q_{2}^{[1]}(x) \text {. } \\
& Y_{0,2}=0, Y_{1,2}=0, \quad Y_{2,2}=1, \quad Y_{3,2}=Q_{1}^{[2]}(x) \text {. } \\
& Y_{0,3}=0, Y_{1,3}=0, \quad Y_{2,3}=0, \quad Y_{3,3}=1 \text {. }
\end{aligned}
$$

Hence is easy to see that the solutions $\left\{Y_{n, 0}\right\}_{n=0}^{\infty},\left\{Y_{n, 1}\right\}_{n=0}^{\infty},\left\{Y_{n, 2}\right\}_{n=0}^{\infty}$, and $\left\{Y_{n, 3}\right\}_{n=0}^{\infty}$ are linearly independent.

THEOREM 2.3. For $i=0,1,2$ or 3 and all $n>2+i$, the following relation holds

$$
Q_{n-i}^{[i]}(x)=R_{n-i}^{[i]}(x)+a_{n} R_{n-i-1}^{[i]}(x)+b_{n} R_{n-i-2}^{[i]}(x),
$$

where

$$
b_{n}=\frac{\left\|Q_{n}\right\|_{S}^{2}}{\left\|R_{n-2}\right\|_{\mu_{2}}^{2}} \neq 0 \quad \text { and } \quad a_{n} \in \mathbb{R} .
$$

Proof. For $i=0,(17)$ is a direct consequence of the orthogonality properties of $Q_{n}$ with respect to $\langle\cdot, \cdot\rangle_{S}$ and $R_{n}$ with respect to $\langle\cdot, \cdot\rangle_{\mu_{2}}$. Hence

$Q_{n}(x)-Q_{n}(t)=\left(R_{n}(x)-R_{n}(t)\right)+a_{n}\left(R_{n-1}(x)-R_{n-1}(t)\right)+b_{n}\left(R_{n-2}(x)-R_{n-2}(t)\right)$. Multiplying both sides of the equation by $\frac{R_{i-1}(t)}{m_{i}(x-t)}$ and integrating on $I$ with respect to the measure $d \mu_{2}(t)$ we obtain (17) for $i=1,2,3$.

COROLlary 2.4. For $i=0,1,2$ or 3 and all $n>2+i$ the following relation holds

$$
Q_{n-i}^{[i]}(x)=\left(x-\widetilde{\eta}_{n-1}\right) R_{n-i-1}^{[i]}(x)-\widetilde{\gamma}_{n-1} R_{n-i-2}^{[i]}(x),
$$

where $\widetilde{\eta}_{n-1}=\eta_{n-1,2}-a_{n}$ and $\widetilde{\gamma}_{n-1}=\gamma_{n-1,2}-b_{n}$. Furthermore, the polynomials $Q_{n-i}^{[i]}$ and $R_{n-i-1}^{[i]}$ are coprime.

Proof. From (11), $R_{n-i}^{[i]}(x)=\left(x-\eta_{n-1,2}\right) R_{n-i-1}^{[i]}(x)-\gamma_{n-1,2} R_{n-i-2}^{[i]}(x)$ and substituting $R_{n-i}^{[i]}(x)$ in (17) we have (19). Note that $\widetilde{\gamma}_{n-1}=\left\|R_{n-2}\right\|_{\mu_{2}}^{-2} C_{n, 1} \neq 0$ and obviously the polynomials $Q_{n-i}^{[i]}$ and $R_{n-i-1}^{[i]}$ are coprime.

THEOREM 2.5. The following relation holds

$$
Q_{n-i-1}^{[i+1]}(x) R_{n-i-1}^{[i]}(x)-Q_{n-i}^{[i]}(x) R_{n-i-2}^{[i+1]}(x)=C_{n, i} \neq 0, \quad n \geq 3,
$$

where $C_{n, i}=\frac{\left\|R_{n-1}\right\|_{\mu_{2}}^{2}-\left\|Q_{n}\right\|_{S}^{2}}{\left\|R_{i}\right\|_{\mu_{2}}^{2}}, i=0,1,2,3$. 
Proof. If $\Lambda_{n, i}=Q_{n-i-1}^{[i+1]}(x) R_{n-i-1}^{[i]}(x)-Q_{n-i}^{[i]}(x) R_{n-i-2}^{[i+1]}(x)$, multiplying (17) for $i+1$ by $R_{n-i-1}^{[i]}(x)$ and (17) by $R_{n-i-2}^{[i+1]}(x)$, taking the difference between these expressions and using (12)

$$
\begin{aligned}
\Lambda_{n, i}= & R_{n-i-1}^{[i+1]}(x) R_{n-i-1}^{[i]}(x)-R_{n-i}^{[i]}(x) R_{n-i-2}^{[i+1]}(x)-b_{n} R_{n-i-2}^{[i]}(x) R_{n-i-2}^{[i+1]}(x) \\
& +b_{n} R_{n-i-3}^{[i+1]}(x) R_{n-i-1}^{[i]}(x)=\prod_{k=i+1}^{n-1} \gamma_{k, 2}-b_{n} \prod_{k=i+1}^{n-2} \gamma_{k, 2} .
\end{aligned}
$$

From (12) and (18), we get the above expression of $C_{n, i}$. Now, suppose that there exists $n_{0} \in \mathbb{N}$ such that $C_{n_{0}, i} \equiv 0$ or, equivalently,

$$
Q_{n_{0}-i-1}^{[i+1]}(x) R_{n_{0}-i-1}^{[i]}(x)=Q_{n_{0}-i}^{[i]}(x) R_{n_{0}-i-2}^{[i+1]}(x)
$$

Taking into account Corollary 2.4 and (11), the polynomial $R_{n_{0}-i-1}^{[i]}$ doesn't have common zeros with the polynomials $R_{n_{0}-i-2}^{[i+1]}$ and $Q_{n_{0}-i}^{[i]}$. Evaluating (21) in any zero of $R_{n_{0}-i-1}^{[i]}$ we have a contradiction. Hence $C_{n, i} \neq 0$.

\section{Zero location and interlacing}

In this section we consider a Sobolev inner product as (7) where the measure $\mu$ is supported on a interval $I=[\alpha, \beta]$ with $\beta \leq 0$. The zero location of orthogonal polynomials with respect to this inner product was studied in $[1,6,10,11]$, where the authors proved that the zeros of the polynomial $Q_{n}$ are real, simple, and at least $n-1$ of them belong to the interior of $I$. We will summarize some of the results of such contributions which will be very useful in the sequel.

Let $\left\{R_{n}(x)\right\}_{n=0}^{\infty}$ be monic orthogonal polynomial sequence with respect to the measure $d \mu_{2}(x)=x^{2} d \mu(x)$, that is,

(i) $\operatorname{deg}\left(R_{n}(x)\right)=n$,

(ii) $\left\langle R_{n}, R_{m}\right\rangle_{\mu_{2}}=\int_{I} R_{n}(x) R_{m}(x) d \mu_{2}(x)=\kappa_{n} \delta_{n, m}$, with $\kappa_{n}>0$ for all $n \in \mathbb{N}$.

Lemma 3.1. [6] Assume that $\beta \leq 0$. If the orthogonality interval I of the monic orthogonal polynomial sequence $\left\{P_{n}\right\}_{n=0}^{\infty}$ is lower bounded then there exists

$$
n_{0} \in \mathbb{N} \quad \text { such that } Q_{n}(0)<0 \quad \forall n \geq n_{0},
$$

and the zeros $x_{n, i}, i=1, \ldots, n$, of $Q_{n}(x)$ satisfy the following separation property:

$$
z_{n-1,1}<x_{n, 1}<z_{n-1,2}<x_{n, 2} \cdots<z_{n-1, n-1}<x_{n, n-1}<\beta \leq 0<x_{n, n}
$$

where $z_{n-1,1}<z_{n-1,2}<\cdots<z_{n-1, n}$ are the zeros of $R_{n-1}$.

LEMma 3.2. [1], [6] If I is a bounded interval and $Q_{n}(0)<0$, then

So, $x_{n, n}$ converges to zero.

$$
0<x_{n, n}<\frac{-\alpha}{n-1} \text {. }
$$

LEMmA 3.3. Let $\left\{Q_{n}(x)\right\}_{n=0}^{\infty}$ be the monic orthogonal polynomial sequence associated with the Sobolev product (7), then

$$
\lambda Q_{n}^{\prime}(0)=-\int_{I} x Q_{n}(x) d \mu(x) .
$$


With these assumptions, we get

THEOREM 3.4. The zeros of the polynomial $Q_{n}^{[i]}$ separate the zeros of the polynomial $Q_{n-1}^{[i+1]}, i=0,1,2$, in the following way

$$
x_{n, 1}^{[i]}<x_{n-1,1}^{[i+1]}<x_{n, 2}^{[i]}<x_{n-1,2}^{[i+1]}<x_{n, 3}^{[i]}<\cdots<x_{n-1, n-1}^{[i+1]}<x_{n, n}^{[i]},
$$

where $x_{n, 1}^{[i]}<x_{n, 2}^{[i]}<\cdots<x_{n, n}^{[i]}$ and $x_{n-1,1}^{[i+1]}<x_{n-1,2}^{[i+1]}<\cdots<x_{n-1, n-1}^{[i+1]}$ are the zeros of $Q_{n}^{[i]}$ and $Q_{n-1}^{[i+1]}$, respectively.

Proof. It is enough to prove the result for $i=0$.

Then from (20) and Lemma 3.1

$$
x_{n, 0}=-1<z_{n-1,1}<x_{n, 1}<z_{n-1,2}<x_{n, 2}<\cdots<z_{n-1, n-1}<0<x_{n, n},
$$

we have that

$$
Q_{n-1}^{[1]}\left(x_{n, k}\right) R_{n-1}\left(x_{n, k}\right)=Q_{n-1}^{[1]}\left(x_{n, k+1}\right) R_{n-1}\left(x_{n, k+1}\right), \quad k=0, \ldots, n-1 .
$$

As it is very well known, $R_{n-1}\left(x_{n, k}\right)$ and $R_{n-1}\left(x_{n, k+1}\right)$ have opposite signs. So, $Q_{n-1}^{[1]}\left(x_{n, k}\right)$ and $Q_{n-1}^{[1]}\left(x_{n, k+1}\right)$ also have opposite signs, then $Q_{n-1}^{[1]}$ has one zero between $x_{n, k}$ and $x_{n, k+1} \cdot Q_{n-1}^{[1]}$ cannot have more than one zero between $x_{n, k}$ and $x_{n, k+1}$. Then we have $(22)$.

\section{Gauss Jacobi Quadrature Formulas}

TheOREM 4.1. Let $Q_{n}(x)$ be the monic orthogonal polynomial of degree $n$ with respect to the Sobolev inner product (7) and $x_{n, 1}<x_{n, 2}<\cdots<x_{n, n}$ its zeros. Then for all polynomial of degree at most $2 n-3, \mathcal{P}_{2 n-3}(x)$, we have

$$
\int_{I} \mathcal{P}_{2 n-3}(x) d \mu_{2}(x)=\sum_{i=1}^{n} \lambda_{n, i} \mathcal{P}_{2 n-3}\left(x_{n, i}\right),
$$

where

$$
\lambda_{n, i}=\int_{I} \frac{Q_{n}(x) d \mu_{2}(x)}{Q_{n}^{\prime}\left(x_{n, i}\right)\left(x-x_{n, i}\right)} .
$$

Proof. Let $\mathcal{L}_{n-1}$ be the Lagrange polynomial that interpolates $\mathcal{P}_{2 n-3}(x)$ in the $n$ zeros of the monic orthogonal polynomial $Q_{n}$, i. e.,

$$
\mathcal{L}_{n-1}(x)=\sum_{i=1}^{n} \mathcal{P}_{2 n-3}\left(x_{n, i}\right) \frac{Q_{n}(x)}{Q_{n}^{\prime}\left(x_{n, i}\right)\left(x-x_{n, i}\right)} .
$$

Then

$$
x^{2} \mathcal{P}_{2 n-3}(x)-x^{2} \mathcal{L}_{n-1}(x)=x^{2} Q_{n}(x) s_{n-3}(x),
$$

where $s_{n-3}$ is a polynomial of degree at most $n-3$. From the orthogonality

Then

$$
\int_{I} x^{2}\left(\mathcal{P}_{2 n-3}(x)-\mathcal{L}_{n-1}(x)\right) d \mu(x)=\int_{I} x^{2} Q_{n}(x) s_{n-3}(x) d \mu(x)=0 .
$$

$$
\int_{I} \mathcal{P}_{2 n-3}(x) d \mu_{2}(x)=\sum_{i=1}^{n} \mathcal{P}_{2 n-3}\left(x_{n, i}\right)\left(\int_{I} \frac{Q_{n}(x) d \mu_{2}(x)}{Q_{n}^{\prime}\left(x_{n, i}\right)\left(x-x_{n, i}\right)}\right) .
$$


Let us consider the particular case, for $k=1, \ldots, n-1$, when

Thus

$$
\mathcal{P}_{2 n-3}(x)=\left(\frac{Q_{n}(x)}{Q_{n}^{\prime}\left(x_{n, k}\right)\left(x-x_{n, k}\right)}\right)^{2} \frac{1}{x-x_{n, n}} .
$$

In other words,

$$
\int_{I}\left(\frac{Q_{n}(x)}{Q_{n}^{\prime}\left(x_{n, k}\right)\left(x-x_{n, k}\right)}\right)^{2} \frac{1}{x-x_{n, n}} d \mu_{2}(x)=\frac{\lambda_{n, k}}{x_{n, k}-x_{n, n}} .
$$

$$
\lambda_{n, k}=\int_{I}\left(\frac{Q_{n}(x)}{Q_{n}^{\prime}\left(x_{n, k}\right)\left(x-x_{n, k}\right)}\right)^{2} \frac{x_{n, k}-x_{n, n}}{x-x_{n, n}} d \mu_{2}(x)>0 ; \quad k=1, \ldots, n-1 .
$$

For $k=n$, let consider the polynomial

We have that

$$
\mathcal{P}_{2 n-3}(x)=\left(\frac{Q_{n}(x)}{Q_{n}^{\prime}\left(x_{n, n}\right)\left(x-x_{n, n}\right)}\right)^{2} \frac{1}{x-x_{n, 1}} .
$$

or, equivalently,

$$
\int_{I}\left(\frac{Q_{n}(x)}{Q_{n}^{\prime}\left(x_{n, n}\right)\left(x-x_{n, n}\right)}\right)^{2} \frac{1}{x-x_{n, 1}} d \mu_{2}(x)=\frac{\lambda_{n, n}}{x_{n, n}-x_{n, 1}},
$$

$$
\lambda_{n, n}=\int_{I}\left(\frac{Q_{n}(x)}{Q_{n}^{\prime}\left(x_{n, n}\right)\left(x-x_{n, n}\right)}\right)^{2} \frac{x_{n, n}-x_{n, 1}}{x-x_{n, 1}} d \mu_{2}(x) .
$$

\section{Markov Theorem}

Proposition 5.1. Let $\left\{Q_{n}(x)\right\}_{n=0}^{\infty}$ be the monic orthogonal polynomial sequence with respect to the Sobolev inner product (7). If $I=[\alpha, \beta], \beta<0$, is bounded, then

$$
\frac{Q_{n-1}^{[1]}(x)}{Q_{n}(x)}=\sum_{k=1}^{n} \frac{\lambda_{n, k}}{\left|\mu_{2}\right|\left(x-x_{n, k}\right)},
$$

where $\lambda_{n, k}$ are the Christoffel coefficients defined by (24), $x_{n, k}$ with $k=1, \ldots, n$, are the zeros of $Q_{n}$, and $\left|\mu_{2}\right|$ is the moment of seconder order with respect to the measure $\mu$.

Proof. $I$ is bounded, then from the previous results we have that the zeros of $Q_{n}$ are simple and at least $n-1$ are in the interior of $I$. Thus

$$
\frac{Q_{n-1}^{[1]}(x)}{Q_{n}(x)}=\sum_{k=1}^{n} \frac{b_{n, k}}{x-x_{n, k}} \quad \text { with } \quad b_{n, k}=\lim _{x \rightarrow x_{n, k}}\left(x-x_{n, k}\right) \frac{Q_{n-1}^{[1]}(x)}{Q_{n}(x)} .
$$

But,

$$
\begin{aligned}
b_{n, k} & =\lim _{x \rightarrow x_{n, k}} \frac{\left(x-x_{n, k}\right)}{Q_{n}(x)} Q_{n-1}^{[1]}(x)=\lim _{x \rightarrow x_{n, k}} \frac{\left(x-x_{n, k}\right)}{Q_{n}(x)} \lim _{x \rightarrow x_{n, k}} Q_{n-1}^{[1]}(x) \\
& =\frac{1}{\left|\mu_{2}\right| Q_{n}^{\prime}\left(x_{n, k}\right)} \int_{I} \frac{Q_{n}(t)}{t-x_{n, k}} t^{2} d \mu(t)=\frac{1}{\left|\mu_{2}\right|} \int_{I} \frac{Q_{n}(t)}{\left(t-x_{n, k}\right) Q_{n}^{\prime}\left(x_{n, k}\right)} t^{2} d \mu(t) \\
& =\frac{\lambda_{n, k}}{\left|\mu_{2}\right|}
\end{aligned}
$$


Proposition 5.2. Under the hypotheses of Proposition 5.1, the sequence of rational functions $\left\{\frac{Q_{n-1}^{[1]}(x)}{Q_{n}(x)}\right\}_{n=1}^{\infty}$ is uniformly bounded in $\mathbb{C} \backslash A$ where $A$ is the convex hull of the set of the zeros of all the polynomials $Q_{n}(x), n>0$.

Proof. First, we prove that $\sum_{k=1}^{n-1} \lambda_{n k} \leq M\left|\mu_{2}\right|$, with $M>1$. For that, we use the Gauss Jacobi Quadrature Formula when $\mathcal{P}_{2 n-3}(x)=-x+x_{n, n}$. Thus,

$$
\begin{aligned}
\underbrace{\int_{I}^{x^{2}\left(-x+x_{n, n}\right) d \mu(x)}}_{>0} & =\sum_{k=1}^{n} \lambda_{n, k}\left(-x_{n, k}+x_{n, n}\right) \\
& =\sum_{k=1}^{n-1} \lambda_{n, k}\left(-x_{n, k}+x_{n, n}\right) .
\end{aligned}
$$

But, by Lemma 3.1,

$$
\sum_{k=1}^{n-1} \lambda_{n, k}\left(-x_{n, k}+x_{n, n}\right) \geq d \sum_{k=1}^{n-1} \lambda_{n, k} \quad \text { with } \quad d=\left|x_{n, n}-\beta\right|
$$

where $\beta$ represents the right end point of $I$. So,

$$
\left.\sum_{k=1}^{n-1} \lambda_{n, k} \leq \frac{1}{d}(\int_{I} x^{2} \underbrace{\left(-x+x_{n, n}\right.}_{\leq d^{\prime}}) d \mu(x)\right)
$$

where $d^{\prime}=\left|x_{n, n}-\alpha\right|$ and $\alpha$ denotes the left end point of $I$.

Then we have that

$$
\sum_{k=1}^{n-1} \lambda_{n k} \leq \frac{d^{\prime}}{d}\left|\mu_{2}\right|
$$

Now, to prove that the sequence $\left\{\frac{Q_{n-1}^{[1]}(x)}{Q_{n}(x)}\right\}_{n=1}^{\infty}$ is uniformly bounded, we choose a compact set $K \subset \mathbb{C} \backslash A$. By Proposition 5.1 we have that

$$
\begin{aligned}
& \left|\frac{Q_{n-1}^{[1]}(x)}{Q_{n}(x)}\right|=\left|\sum_{k=1}^{n} \frac{\lambda_{n, k}}{\left|\mu_{2}\right|\left(x-x_{n, k}\right)}\right| \leq\left|\sum_{k=1}^{n-1} \frac{\lambda_{n, k}}{\left|\mu_{2}\right|\left(x-x_{n, k}\right)}\right|+\left|\frac{\lambda_{n, n}}{\left|\mu_{2}\right|\left(x-x_{n, n}\right)}\right| \\
& \leq \frac{1}{D\left|\mu_{2}\right|}\left(2 \sum_{k=1}^{n-1} \lambda_{n, k}-\left|\mu_{2}\right|\right) \leq \frac{1}{D}\left(2 \frac{d^{\prime}}{d}-1\right), \quad \text { where } \quad D=\min _{\substack{x \in A \\
y \in K}}|x-y| .
\end{aligned}
$$

Notice that $D>0$ since $K$ is a compact set and $A$ is a closed set.

Definition 5.3. Let $\mu$ be a positive Borel measure supported on I. The Markov type function associated with $\mu$ is the function

$$
\widetilde{\mu}(x)=\frac{1}{\left|\mu_{2}\right|} \int_{I} \frac{t^{2} d \mu(t)}{x-t} .
$$

The next result gives a relation between the Markov type functions, the monic orthogonal polynomials with respect to the Sobolev inner product (7) and its associated polynomials of the first kind for $i=1$. 
Proposition 5.4. Let $\mu$ be a positive Borel measure supported on $I$ and $Q_{n}(x)$ and $Q_{n-1}^{[1]}(x)$ defined as above. Then

$$
\widetilde{\mu}(x)-\frac{Q_{n-1}^{[1]}(x)}{Q_{n}(x)}=\frac{1}{\left|\mu_{2}\right|} \int_{I} \frac{Q_{n}(t)}{Q_{n}(x)} \frac{t^{2} d \mu(t)}{x-t}=\mathcal{O}\left(\frac{1}{x^{2 n-1}}\right) .
$$

Proof. Using the definition of $Q_{n-1}^{[1]}(x)$, we have

Thus,

$$
\begin{aligned}
Q_{n-1}^{[1]}(x) & =\frac{1}{\left|\mu_{2}\right|} \int_{I} \frac{Q_{n}(x)-Q_{n}(t)}{x-t} t^{2} d \mu(t) \\
& =Q_{n}(x) \frac{1}{\left|\mu_{2}\right|} \int_{I} \frac{t^{2}}{x-t} d \mu(t)-\frac{1}{\left|\mu_{2}\right|} \int_{I} \frac{Q_{n}(t) t^{2}}{x-t} d \mu(t) \\
& =Q_{n}(x) \widetilde{\mu}(x)-\frac{1}{\left|\mu_{2}\right|} \int_{I} \frac{Q_{n}(t) t^{2}}{x-t} d \mu(t) .
\end{aligned}
$$

$$
\frac{Q_{n-1}^{[1]}(x)}{Q_{n}(x)}=\widetilde{\mu}(x)-\frac{1}{\left|\mu_{2}\right|} \int_{I} \frac{Q_{n}(t)}{Q_{n}(x)} \frac{t^{2} d \mu(t)}{x-t} .
$$

From this last relation we have the first equality. To obtain the second one we use the orthogonality condition

From this we get

$$
\left\langle Q_{n}(t), \frac{\left(Q_{n-2}(x)-Q_{n-2}(t)\right) t^{2}}{\left|\mu_{2}\right|(x-t)}\right\rangle_{S}=0 .
$$

$$
\begin{aligned}
\int_{I} Q_{n}(t) \frac{Q_{n-2}(x)-Q_{n-2}(t)}{\left|\mu_{2}\right|(x-t)} t^{2} d \mu(t) & =0 \\
\frac{1}{\left|\mu_{2}\right|} \int_{I} \frac{Q_{n}(t) Q_{n-2}(x) t^{2}}{x-t} d \mu(t) & =\frac{1}{\left|\mu_{2}\right|} \int_{I} \frac{Q_{n}(t) Q_{n-2}(t) t^{2}}{x-t} d \mu(t) \\
\frac{1}{\left|\mu_{2}\right|} \int_{I} \frac{Q_{n}(t)}{Q_{n}(x)} \frac{t^{2}}{x-t} d \mu(t) & =\frac{1}{\left|\mu_{2}\right|} \int_{I} \frac{Q_{n}(t) Q_{n-2}(t)}{Q_{n}(x) Q_{n-2}(x)} \frac{t^{2}}{x-t} d \mu(t) .
\end{aligned}
$$

TheOREM 5.5. (Markov's Theorem) Let assume that I is a compact set. Then the sequence of rational functions $\left\{\frac{Q_{n-1}^{[1]}(x)}{Q_{n}(x)}\right\}_{n=1}^{\infty}$ is uniformly convergent to $\widetilde{\mu}$ in every compact subset of $\overline{\mathbb{C}} \backslash A$ where $A$ is convex hull of the set of the zeros of $Q_{n}(x)$ for every $n>0$.

Proof. From the second equality in Proposition 5.4 we have that

Let us consider

$$
\widetilde{\mu}(z)-\frac{Q_{n-1}^{[1]}(z)}{Q_{n}(z)}=O\left(\frac{1}{z^{2 n-1}}\right), \quad z \rightarrow \infty .
$$

$$
l_{\rho}=\{z:|\varphi(z)|=\rho\},
$$

where $\varphi$ is the conformal representation of $\overline{\mathbb{C}} \backslash A$ onto $\{w \in \mathbb{C}:|w|>1\}$ such that $\varphi(\infty)=\infty, \quad \varphi^{\prime}(\infty)>0$. Of course, 


$$
\frac{\widetilde{\mu}(z)-\frac{Q_{n-1}^{[1]}(z)}{Q_{n}(z)}}{\varphi^{2 n-1}(z)} \in H(\mathbb{C} \backslash A),
$$

since $\varphi$ has a simple zero at $z=\infty$. On the other hand,

$$
\sup _{z \in l_{\rho}}\left|\frac{\widetilde{\mu}(z)-\frac{Q_{n-1}^{[1]}(z)}{Q_{n}(z)}}{\varphi^{2 n-1}(z)}\right| \leq \frac{C_{l_{\rho}}}{\rho^{2 n-1}},
$$

since the sequence $\left\{\frac{Q_{n-1}^{[1]}(z)}{Q_{n}(z)}\right\}_{n=1}^{\infty}$ is uniformly bounded over compact subsets of $\overline{\mathbb{C}} \backslash A$. According to the maximum principle, the function is bounded in every disk $D_{\rho}=\{z:|\varphi(z)|<\rho\}$.

Let $K$ be any compact set on $\overline{\mathbb{C}} \backslash A$. Taking $\rho$ close enough to 1 , we have that $K \subset D_{\rho}$ and, then, for $z \in K$

$$
\begin{aligned}
\sup _{z \in K}\left|\widetilde{\mu}(z)-\frac{Q_{n-1}^{[1]}(z)}{Q_{n}(z)}\right| & \leq \frac{C_{l_{\rho}}}{\rho^{2 n-1}} \sup _{z \in K}|\varphi(z)|^{2 n-1} \\
& =C_{l_{\rho}}\left(\frac{\|\varphi\|_{K}}{\rho}\right)^{2 n-1} \underset{n \rightarrow \infty}{\longrightarrow} 0
\end{aligned}
$$

which proves the statement.

We shall notice that we can estimate the speed of convergence in the above rational approximation.

Indeed, taking the $2 n$th root in (25) we get

$$
\limsup _{n}\left\|\widetilde{\mu}(z)-\frac{Q_{n-1}^{[1]}(z)}{Q_{n}(z)}\right\|_{K}^{1 / 2 n} \leq \frac{\|\varphi\|_{K}}{\rho}
$$

for all $\rho$ sufficiently close to 1 . Finally, taking into account that $\rho$ converges to 1 , we obtain

$$
\limsup _{n}\left\|\widetilde{\mu}(z)-\frac{Q_{n-1}^{[1]}(z)}{Q_{n}(z)}\right\|_{K}^{1 / 2 n} \leq\|\varphi\|_{K}<1
$$

\section{Acknowledgements}

The authors thank the careful work done by the referees. Their comments and suggestions have contributed to improve substantially the presentation.

\section{References}

[1] M. Alfaro, F. Marcellán, M. L. Rezola, and A. Ronveaux, On orthogonal polynomials of Sobolev type: Algebraic properties and zeros, SIAM J. Math. Anal. 23 (1992), 


\section{REFERENCES}

$737-757$.

[2] A. J. Durán, A generalization of Favard's theorem for polynomials satisfying a recurrence relation, J. Approx. Theory 74 (1993), 83-109.

[3] A. J. Durán and W. Van Assche, Orthogonal matrix polynomials and higher-order recurrence relations, Linear Algebra Appl. 219 (1995), 261-280.

[4] D. Evans L. L. Littlejohn, F. Marcellán, C. Markett, and A. Ronveaux, On recurrence relations for Sobolev polynomials, SIAM J. Math. Anal. 26 (1995,) 446-467.

[5] F. Marcellán and R. Alvarez-Nodarse, On the Favard Theorem and their extensions. J. Comput. Appl. Math. 127 (2001), 231-254.

[6] F. Marcellán, T. E. Pérez, and M. A. Piñar On zeros of Sobolev-Type orthogonal polynomials, Rendi. di. Mat. Roma (serie VII) 12, (1992), 455-473.

[7] F. Marcellán and A. Ronveaux, On a class of polynomials orthogonal with respect to a discrete Sobolev inner product, Indag. Math. (N. S.) 1 (1990), 451-464.

[8] F. Marcellán and S. M. Zagorodnyuk, On the basic set of solutions of a high-order linear difference equation, J. Diff. Eq. Appl. 12 (2006), 213-228.

[9] P. Nevai and W. Van Assche, Compact perturbations of orthogonal polynomials, Pac. J. of Math. 153, No. 1, (1992), 163-184.

[10] T. E. Pérez and M. A. Piñar Global Properties of zeros for Sobolev-Type Orthogonal Polynomials, J. Comput. Appl. Math. 4 (1993), 225-232.

[11] M. A. Piñar, Polinomios Ortogonales de tipo Sobolev. Aplicaciones, Ph.D Thesis, Universidad de Granada, 1992. In Spanish.

[12] W. Van Assche, Orthogonal polynomials, associated polynomials and functions of the second kind, J. Comput. Appl. Math. 37 (1991), 237-249. 